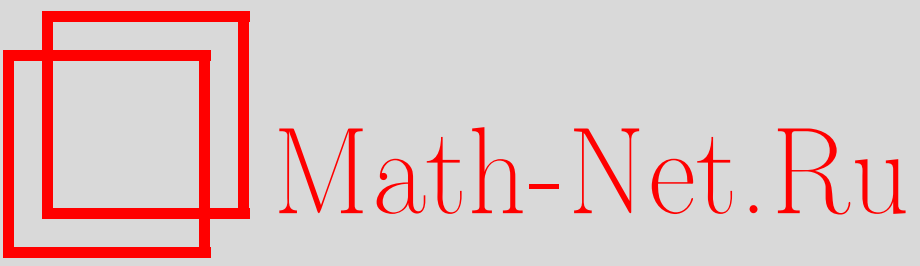

А. Ю. Черный, Влияние критических флуктуаций при структурном фазовом переходе на сверхпроводящее спаривание, ТМФ, 1996, том 107, номер 1, 162 173

DOI: https://doi.org/10.4213/tmf1146

Использование Общероссийского математического портала Math-Net.Ru подразумевает, что вы прочитали и согласны с пользовательским соглашением http://www . mathnet.ru/rus/agreement

Параметры загрузки:

IP : 52.90 .164 .192

26 апреля 2023 г., 13:52:08 


\title{
ВЛИЯНИЕ КРИТИЧЕСКИХ ФЛУКТУАЦИЙ ПРИ СТРУКТУРНОМ ФАЗОВОМ ПЕРЕХОДЕ НА СВЕРХПРОВОДЯШЕЕ СПАРИВАНИЕ
}

\begin{abstract}
В рамках ангармонической модели высокотемпературной сверхпроводимости исследуется влияние критических флуктуаций в окрестности структурного фазового перехода (СФП) на изотопический эффект (ИЭ). Показывается, что при совпадении температур структурного и сверхпроводящего переходов ИЭ определяется критическим индексом $\gamma$. В случае, когда $\gamma<1$, фактор ИЭ для температуры сверхпроводящего перехода $T_{c}\left(\alpha=-\frac{\partial \ln T_{c}}{\partial \ln M}\right)$ совпадает с фактором ИЭ для температуры структурного перехода $T_{0}$ (т.е. $\alpha=\varepsilon$, где $\varepsilon=-\frac{\partial \ln T_{0}}{\partial \ln M}$ ), а в случае $\gamma>1$ фактор $\alpha=\frac{1}{2}$ (при пренебрежении кулоновским отталкиванием). Этот результат носит общий характер и не зависит от выбора модели СФП. Результат демонстрируется на примере точно решаемой модели С $\Phi П$ с $\gamma=2$.
\end{abstract}

\section{1. ВВЕДЕНИЕ}

Исследованию изотопического эффекта (ИЭ) в новых оксидных сверхпроводниках посвяшено большое количество работ (см. обзор [1]). Значение ИЭ в высокотемпературных сверхпроводниках (ВТСП) сильно зависит от концентрации примесей допирующих элементов, возрастая по мере удаления от их оптимальных значений, обеспечивающих максимально высокие $T_{c}$. При этом фактор ИЭ может возрастать до аномально высоких значений, превышаюших 0,5 , что необъяснимо в рамках теории БКШ.

Такое аномальное поведение ИЭ по кислороду в ВТСП представляет значительньй интерес для понимания микроскопического механизма сверхпроводимости, т.к. позволяет глубже понять роль динамики решетки в этом механизме. Предложено несколько объяснений аномального поведения ИЭ в рамках различных механизмов высокотемпературной сверхпроводимости [1].

В рамках ангармонической модели для ВТСП, предложенной Н. М. Плакидой с сотрудниками [2], удается объяснить не только значения $\alpha>\frac{1}{2}$, но и резкий скачок ИЭ, наблюдаемый в соединениях $\mathrm{La}_{2-x} M_{x} \mathrm{CuO}_{4}(M=\mathrm{Ba}, \mathrm{Sr})$ на границе структурного фазового перехода (СФП) [3,4]. Однако вычисления восприимчивости для динамики решетки проводились в этих работах в приближении среднего поля, что не позволяло исследовать область критических флуктуаций в окрестности температуры СФП.

В этой работе рассматривается электрон-фононная система с точно решаемой решеточной частью. Асимптотически точная решаемость модели СФП, изученной в $[5,6]$, позволяет исследовать влияние СФП на ИЭ в области критических фллуктуаций для 
СФП. Отметим, что такое исследование, проведенное автором (совместно с Н. М. Плакидой) в работе [7], не совсем точно учитывает влияние критических флуктуаций на ИЭ.

При расчетах мы считаем, что СФП обусловлен ангармоническим ион-ионным потенциалом, и поэтому мы пренебрегаем влиянием электронов на динамику решетки. При этих условиях в рамках стандартной теории Элиашберга получается обший результат: ИЭ при совпадении критических температур структурного и сверхпроводяще20 переходов определяется критическим индексом $\gamma\left(\chi^{-1}\left(q=q_{1}, \omega=0\right) \sim \tau^{\gamma}, \chi-\right.$ статистическая фононная восприимчивость, $q_{1}-$ вектор обратной решетки для мягкой моды колебаний, $\tau=\frac{T-T_{0}}{T_{0}}-$ приведенная температура). Если $\gamma<1$, то фактор ИЭ $\alpha=\varepsilon$, где $\varepsilon=-\frac{\partial \ln T_{0}}{\partial \ln M}-$ фактор ИЭ для температуры СФП. В случае $\gamma>1$ имеем $\alpha=\frac{1}{2}$ (если постоянная кулоновского взаимодействия $\mu^{*}=0$ ).

В разделе 2 приводятся гамильтониан электрон-фононной системы и выражение для температуры сверхпроводящего перехода в рамках теории Элиашберга, обобшенной на случай ангармонических колебаний решетки. Здесь же рассматривается вклад мягкой моды колебаний в постоянную электрон-фононной связи $\lambda$. Раздел 3 посвящен исследованию свойств решетки при структурном переходе и вычислению ее восприимчивости, необходимой для расчета постоянной электрон-фононного взаимодействия. В четвертом разделе вычисляется ИЭ для исходной модели, а также влияние СФП на ИЭ в области критических флуктуаций в зависимости от $\gamma$.

\section{2. ГАМИЛЬТОНИАН МОДЕЛИ. ТЕМПЕРАТУРА СВЕРХПРОВОДЯШЕГО ПЕРЕХОДА}

Рассмотрим электрон-фононную систему с линейным электрон-фононным взаимодействием, описываемую гамильтонианом

$$
H=\sum_{p \sigma} \varepsilon_{p} a_{p \sigma}^{+} a_{p \sigma}+\sum_{q p \sigma} v(q) a_{p \sigma}^{+} a_{p-q \sigma} Q_{q}+H_{i}
$$

здесь $\varepsilon_{p}$ - электронньй ферми-жидкостньй спектр, $v(q)=\frac{i q V(q)}{\sqrt{M}}$, где $V(q)$ - матричный элемент экранированного электрон-фононного взаимодействия, $M$ - масса иона, $Q_{q}$ - нормальная фононная переменная, $H_{i}$ - энергия колебаний решетки, которая будет рассмотрена в следующем разделе.

При описании электронной части гамильтониана мы не рассматриваем прямого кулоновского взаимодействия между электронами, полагая, что оно может быть учтено стандартными методами [8] с помощью перенормировки постоянной электрон-фононного взаимодействия $\lambda$ на величину $\mu^{*}$.

В приближении Мигдала-Элиашберга, обобщенном на случай произвольных колебаний решетки (см. [9]), для постоянной электрон-фононного взаимодействия $\lambda$, определяюшей температуру сверхпроводяшего перехода в системе, получаем обычное выражение

$$
\lambda=\int_{-\infty}^{+\infty} d \omega \frac{\alpha^{2} F(\omega)}{\omega}
$$

где

$$
\alpha^{2} F(\omega)=\frac{1}{N(0)} \int_{s_{f}} \frac{d^{2} p}{v_{p}} \int_{s_{f}} \frac{d^{2} p^{\prime}}{v_{p^{\prime}}}\left[-\frac{1}{\pi} \operatorname{Im}\left\langle\left\langle Q_{q} \mid Q_{-q}\right\rangle\right\rangle_{\omega+i \varepsilon}\right]\left|v\left(p-p^{\prime}\right)\right|^{2} .
$$


Здесь $N(0)$ - плотность электронных состояний на поверхности Ферми $s_{f}, \quad v_{p}=$ $\left|\vec{\nabla}_{p} \varepsilon_{p}\right|$ - групповая скорость электронов на поверхности Ферми $s_{f}$, введена функция $\left\langle\left\langle Q_{q} \mid Q_{-q}\right\rangle\right\rangle_{\omega+i \varepsilon}=-\chi_{q}(\omega)$, являюшаяся фурье-образом временной запаздываюшей функции Грина [10] и с точностью до знака совпадаюшая с комплексной фононной восприимчивостью.

Воспользовавшись дисперсионными соотношениями для функций Грина [10], после интегрирования по частоте в (1) получаем

$$
\lambda=\frac{1}{N(0)} \int_{s_{f}} \frac{d^{2} p}{v_{p}} \int_{s_{f}} \frac{d^{2} p^{\prime}}{v_{p^{\prime}}} \frac{|V(q) q|^{2}}{M} \chi_{q}(0),
$$

где $q=p-p^{\prime}, \chi_{q}(0)=-\left\langle\left\langle Q_{q} \mid Q_{-q}\right\rangle\right\rangle_{0+i \varepsilon}-$ статическая восприимчивость решетки.

В случае изотропной поверхности Ферми для оценки постоянной (2) можно использовать приближение Мак-Миллана [11], согласно которому

$$
\lambda=\frac{N(0) J^{2}}{M} \frac{1}{N} \sum_{q} \chi_{q}(0)
$$

где $J^{2}$ - усредненный по поверхности Ферми квадрат абсолютного значения матричного элемента электрон-фононного взаимодействия $|V(q) q|^{2}$, а суммирование по $q$ производится по зоне Бриллюэна.

Необходимо отметить, однако, что переход от выражения (2) к (3) приводит к изменению размерности интегрирования: от четырехмерного в $(2)$ к трехмерному в (3). Поскольку мы интересуемся вкладом мягкой моды колебаний в постоянную $\lambda$, на этом необходимо остановиться более подробно.

Наличие мягких оптических мод колебаний с волновым вектором $q_{1}$ в фононном спектре приводит в общем случае к следующему виду статической восприимчивости в окрестности $q_{1}$ (см., например, [12]):

$$
\chi_{q}(0)=\frac{1}{\Omega_{0}^{2}+c^{2}\left(q-q_{1}\right)^{2}},
$$

где $\Omega_{0}^{2}$ - зависящая от температуры щель в фононном спектре, которая обрашается в нуль при температуре СФП.

В общем случае при $q_{1} \neq 0$ матричный элемент $\left|V\left(q_{1}\right) q_{1}\right| \neq 0$, поэтому для вклада мягкой моды в $\lambda$ получаем

$$
\Delta \lambda \sim \int d^{2} p d^{2} p^{\prime} \frac{1}{\Omega_{0}^{2}+c^{2}\left(p-p^{\prime}-q_{1}\right)^{2}},
$$

где интегрирование проводится в окрестности $q_{1}$.

Размерность участка интегрирования $q_{1}=p-p^{\prime}$, вообше говоря, одномерна, т.к. двумерные поверхности Ферми, сдвинутые на величину $q_{1}$, пересекаются вдоль одномерной кривой. Переходя к новым переменным интегрирования вдоль этой кривой $\left(p_{1}\right)$ и перпендикулярно к ней $\left(p_{\mathrm{tr}}\right)$, получаем

$$
\begin{aligned}
\Delta \lambda & \sim \int d p_{l} d^{3} p_{\operatorname{tr}} \frac{1}{\Omega_{0}^{2}+c^{2}\left(p-p^{\prime}-q_{1}\right)^{2}} \sim \\
& \sim \int d p_{l} d^{3} p_{\operatorname{tr}} \frac{1}{\Omega_{0}^{2}+c^{\prime 2} p_{\operatorname{tr}}^{2}} \sim \int d^{3} p_{\operatorname{tr}} \frac{1}{\Omega_{0}^{2}+c^{\prime 2} p_{\operatorname{tr}}^{2}} .
\end{aligned}
$$


Таким образом, усреднение по трехмерной зоне Бриллюэна (3) приводит качественно к тем же результатам, что и двойное усреднение по поверхности Ферми (2).

Если считать выражение для восприимчивости (4) верным не только в окрестности $q_{1}$, но и во всей зоне Бриллюэна (дебаевское приближение), то после подстановки его в (3) получаем

$$
\lambda=\lambda_{0}\left(1-\frac{\Omega_{0}}{\omega_{0}} \operatorname{arctg} \frac{\omega_{0}}{\Omega_{0}}\right),
$$

где $\lambda_{0}=3 \frac{N(0) J^{2}}{M \omega_{0}^{2}}-$ значение постоянной электрон-фононной связи при $\Omega_{0}=0, \omega_{0}=c q_{0}$ - дебаевский радиус.

Существенно, что выражение для $\lambda(5)$ линейно по $\Omega_{0}$ в первом порядке по $\Omega_{0}$. Этот эффект вклада мягкой моды в постоянную электрон-фононной связи является следствием трехмерности интегрирования в выражении (3) и имеет место в обшем случае независимо от выбора конкретной модели СФП.

Для того чтобы правильно учесть вклад мягкой моды колебаний в выражение для температуры сверхпроводящего перехода, воспользуемся строгими результатами. Как можно показать, точное решение для $T_{c}$ из линеаризованных уравнений Элиашберга имеет вид [13]

$$
\begin{aligned}
T_{c}=\alpha_{0}( & \left.\lambda\left\langle\omega^{2}\right\rangle\right)^{\frac{1}{2}}\left[1+\alpha_{1} \frac{1}{\lambda\left\langle\omega^{2}\right\rangle} \frac{\left\langle\omega^{4}\right\rangle}{\left\langle\omega^{2}\right\rangle}+\right. \\
& \left.+\frac{1}{\left(\lambda\left\langle\omega^{2}\right\rangle\right)^{2}}\left(\alpha_{21} \frac{\left\langle\omega^{6}\right\rangle}{\left\langle\omega^{2}\right\rangle}+\alpha_{22}\left(\frac{\left\langle\omega^{4}\right\rangle}{\left\langle\omega^{2}\right\rangle}\right)^{2}\right)\right]+\ldots
\end{aligned}
$$

где $\langle g(\omega)\rangle=\frac{1}{\lambda} \int_{-\infty}^{+\infty} d \omega g(\omega) \frac{\alpha^{2} F(\omega)}{\omega}-$ среднее по распределению $\frac{\alpha^{2} F(\omega)}{\omega}$, при этом величина, обратная постоянной электрон-фононной связи (1), играет роль нормировочного множителя.

Коэффищиенты $\alpha$ зависят только от эффективной постоянной кулоновского взаимодействия $\mu^{*}$, но не от формы спектра $\alpha^{2} F(\omega)$. При этом разложение (6) сходится по $\lambda$ при $\lambda \geq \Lambda$, где $\Lambda$ - некоторая постоянная, зависяшая от формы спектра и для металлических сверхпроводников обычно лежащая в пределах $1<\Lambda<3$. Как можно строго показать, это выражение в принципе может быть аналитически продолжено в область малых $\lambda$, хотя на практике это довольно трудно осуществить.

Для изучения ИЭ (см. ниже раздел 4) важно выяснить, какую минимальную степень разложения по $\Omega_{0}$ при малых $\Omega_{0}$ содержит правая часть равенства (6). Несмотря на то что выражение для $\lambda(5)$ линейно по $\Omega_{0}$, разложение $T_{c}$ по $\Omega_{0}$ квадратично при малых $\Omega_{0}$.

Действительно, как легко видеть, $\lambda$ входит в выражение (6) только в комбинации с множителем $\left\langle\omega^{2}\right\rangle$, а в выражение для отношения моментов $\frac{\left\langle\omega^{2 n}\right\rangle}{\left\langle\omega^{2}\right\rangle}$ постоянная $\lambda$ вообше не входит. При этом для средних значений (как показано выше на примере $\lambda$ ) может быть использовано приближение Мак-Миллана:

$$
\langle g(\omega)\rangle=\frac{1}{\lambda} \int_{-\infty}^{+\infty} d \omega g(\omega) \frac{\alpha^{2} F(\omega)}{\omega}=\frac{1}{\lambda} \frac{N(0) J^{2}}{M} \frac{1}{N} \sum_{q} \frac{g\left(\Omega_{q}\right)}{\Omega_{q}^{2}}
$$

где $\Omega_{q}$ - перенормированный фононный спектр. 
Отсюда находим, используя приближение Дебая,

$$
\lambda\left\langle\omega^{2}\right\rangle=\frac{N(0) J^{2}}{M}
$$

и

$$
\frac{\left\langle\omega^{2 n}\right\rangle}{\left\langle\omega^{2}\right\rangle}=\frac{3}{\omega_{0}^{3}} \int_{0}^{\omega_{0}} d \omega\left(\Omega_{0}^{2}+\omega^{2}\right)^{n-1} .
$$

Последнее выражение квадратично по $\Omega_{0}$ (при малых $\Omega_{0}$ ) при $n \geq 2$, что доказывает наше утверждение.

Поскольку явного точного выражения для температуры сверхпроводящего перехода не существует, мы будем использовать предложенное Комбескотом очень удачное аппроксимирующее выражение для $T_{c}$, которое обладает отмеченной вьше качественной особенностью и дает отклонение от точного значения в пределах $1 \%$ точности при $\mu^{*}=0[14]$ :

$$
1=\int_{0}^{+\infty} d \omega \frac{\alpha^{2} F(\omega)}{\omega} \ln \left[1+\left(\frac{\omega}{a T_{c}}\right)^{2}\right]
$$

где $a=3,9$.

В приближении Мак-Миллана уравнение на $T_{c}(7)$ принимает вид

$$
1=\frac{1}{2} \frac{N(0) J^{2}}{M} \frac{1}{N} \sum_{q} \frac{1}{\Omega_{q}^{2}} \ln \left[1+\left(\frac{\Omega_{q}}{a T_{c}}\right)^{2}\right] .
$$

Таким образом, для дальнейшего исследования необходимо получить конкретное выражение квазифононного спектра $\Omega_{q}$ в рамках точно решаемой модели С $\Phi$ П.

\section{3. СТРУКТУРНЫЙ ФАЗОВЫЙ ПЕРЕХОД В РЕШЕТКЕ}

Рассмотрим модель СФП, описьваемую решеточным гамильтонианом [15],

$$
H_{i}=\sum_{l} \frac{1}{2 M} p_{l}^{2}+U\left(\left\{X_{l}\right\}\right)
$$

где $U\left(\left\{X_{l}\right\}\right)$ - потенциальная энергия ионов решетки:

$$
U\left(\left\{X_{l}\right\}\right)=\sum_{l}-\frac{1}{2} A X_{l}^{2}+\frac{1}{4} \sum_{l l^{\prime}} \Phi\left(l-l^{\prime}\right)\left(X_{l}-X_{l^{\prime}}\right)^{2}+\frac{B}{4} \frac{1}{N}\left(\sum_{l} X_{l}^{2}\right)^{2} .
$$

Здесь $X_{l}$ и $P_{l}$ соответственно локальные нормальные координата (ЛНК) и импульс для мягкой фононной моды колебаний в $l$-й примитивной ячейке, $A>0$ - параметр, определяюший частоту неустойчивой в гармоническом приближении моды колебаний $\nu_{0}^{2}=-\frac{A}{M}, B$ - постоянная, определяюшая интенсивность ангармонического взаимодействия, $M$ - эффективная масса, пропорциональная массе атома, $\Phi\left(l-l^{\prime}\right)$ - атомная силовая матрица второго порядка.

Сразу отметим, что в этой модели мягкая мода находится в центре зоны Бриллюэна $(\vec{q}=0)$, однако с помощью выбора постоянных гармонического взаимодействия $A$ 
и $\Phi\left(l-l^{\prime}\right)$ мы всегда можем добиться того, чтобы мягкая мода лежала на границе зоны Бриллюэна $(\vec{q} \neq 0)$. Фактически эта процедура - просто сдвиг фононных частот в $\vec{q}$-пространстве на величину $\vec{q}_{1}$, и на рассматриваемые физические свойства системы она не влияет. Поэтому мы будем использовать эту модель СФП, подразумевая, что полученные результаты справедливы для мягкой моды, лежащей на границе зоны Бриллюэна.

Для вычисления спектра квазифононных возбуждений $\Omega_{q}$ воспользуемся методом самосогласованных

фононов [16]. В соответствии с этим методом вычисляем среднее значение потенциальной энергии по аппроксимирующему гамильтониану с псевдогармоническим взаимодействием, представляя локальную нормальную координату в виде

$$
X_{l}=x_{l}+\left\langle X_{l}\right\rangle=x_{l}+c
$$

где $x_{l}$ - оператор смещения ЛНК из положения равновесия, а среднее значение ЛнК $\left\langle X_{l}\right\rangle=c$ не зависит от $l$, если волновой вектор для мягкой моды равен нулю.

Подставляя оператор (11) в выражение (10) и проводя усреднение по псевдогармоническому гамильтониану, получаем для среднего значения потенциальной энергии решетки

$$
\begin{aligned}
\langle U\rangle= & \frac{1}{2} \sum_{l l^{\prime}}\left(\Phi(0)-A+B c^{2}\right)\left\langle x_{l} x_{l^{\prime}}\right\rangle \delta_{l l^{\prime}}+\sum_{l l^{\prime}}\left(-\frac{1}{2} \Phi\left(l-l^{\prime}\right)+\frac{B c^{2}}{N}\right)\left\langle x_{l} x_{l^{\prime}}\right\rangle+ \\
& +\frac{B 1}{4 N}\left(\sum_{l}\left\langle x_{l}^{2}\right\rangle\right)^{2}+\frac{B 1}{2 N} \sum_{l l^{\prime}}\left\langle x_{l} x_{l^{\prime}}\right\rangle^{2}+N\left(-\frac{A}{2} c^{2}+\frac{B}{4} c^{4}\right),
\end{aligned}
$$

где обозначено $\Phi(0)=\Phi(\vec{q}=0)=\sum_{l} \Phi(l)$. При вычислении средних от слагаемых четвертой степени мы воспользовались теоремой Вика.

Силовую матрицу псевдогармонического взаимодействия находим стандартным способом

$$
\begin{aligned}
\tilde{\Phi}\left(l-l^{\prime}\right)= & 2 \frac{\delta\langle U\rangle}{\delta\left\langle x_{l} x_{l^{\prime}}\right\rangle}=\left(\Phi(0)-A+B\left(c^{2}+\left\langle x_{l}^{2}\right\rangle\right)\right) \delta_{l l^{\prime}}- \\
& -\Phi\left(l-l^{\prime}\right)+2 \frac{B}{N}\left(c^{2}+\left\langle x_{l} x_{l^{\prime}}\right\rangle\right) .
\end{aligned}
$$

Учитывая, что в статистическом пределе $N \rightarrow+\infty$ и $\frac{N}{V}=$ const последнее слагаемое асимптотически равно нулю, а $\left\langle x_{l}^{2}\right\rangle=\left\langle x^{2}\right\rangle$ не зависит в силу трансляционной инвариантности от номера ячейки $l$, получаем

$$
\tilde{\Phi}\left(l-l^{\prime}\right)=\left(\Phi(0)-A+B\left(c^{2}+\left\langle x_{l}^{2}\right\rangle\right)\right) \delta_{l l^{\prime}}-\Phi\left(l-l^{\prime}\right) .
$$

Отсюда находим спектр квазигармонических возбуждений

$$
\Omega_{q}^{2}=\frac{\tilde{\Phi}(q)}{M}=-\frac{A}{M}+\frac{\Phi(0)-\Phi(q)}{M}+\frac{B}{M}\left(c^{2}+\left\langle x^{2}\right\rangle\right) \simeq \Omega_{0}^{2}+c^{2} q^{2},
$$

где $\Phi(q)=\sum_{l} \Phi(l) e^{-i q l}$ и

$$
\Omega_{0}^{2}=\Omega_{q}^{2}(q=0)=-\frac{A}{M}+\frac{B}{M}\left(c^{2}+\left\langle x^{2}\right\rangle\right)
$$


- квадрат мягкой моды колебаний.

Параметр порядка с определяется из условия минимума свободной энергии. Пренебрегая асимптотически малыми членами, получаем

$$
\frac{\partial F^{\mathrm{app}}}{\partial c}=\frac{\partial\langle U\rangle}{\partial c}=N c\left(B\left(c^{2}+\left\langle x^{2}\right\rangle\right)-A\right)=0 .
$$

Используя условие устойчивости $\frac{\partial^{2} F^{\text {app }}}{\partial^{2} c}=\frac{\partial^{2}\langle U\rangle}{\partial^{2} c}>0$, находим

$$
\begin{array}{lll}
c=0 & \text { при } & \left\langle x^{2}\right\rangle>\frac{A}{B}, \\
c=\frac{A}{B}-\left\langle x^{2}\right\rangle & \text { при } & \left\langle x^{2}\right\rangle<\frac{A}{B} .
\end{array}
$$

Таким образом, условие $\left\langle x^{2}\right\rangle=\frac{A}{B}$ определяет температуру СФП $T_{0}$, ниже которой среднее значение ЛНК отлично от нуля.

Из уравнений (15) и (16) получаем самосогласованное уравнение для мягкой моды в высокосимметричной фазе $(c=0)$, учитывая, что $\left\langle x^{2}\right\rangle=\frac{1}{M} \sum_{q}\left\langle Q_{-q} Q_{q}\right\rangle$ :

$$
\Omega_{0}^{2}+\frac{A}{M}=\frac{B}{M^{2}} \frac{1}{N} \sum_{q} \frac{1}{2 \Omega_{q}} \operatorname{cth} \frac{\Omega_{q}}{2 T} .
$$

При температуре СФП $T_{0}$ мягкая мода (15), которая есть щель в фононном спектре колебаний решетки, обращается в нуль и в низкосимметричной фазе $(c \neq 0)$ при всех $T<T_{0}$ остается равной 0 за счет отличного от нуля среднего значения смешения ионов, что является аномальным свойством рассматриваемой модели СФП, возникающим вследствие асимптотической малости последнего слагаемого в выражении (13).

Статическая восприимчивость связана со спектром перенормированных фононных возбуж дений стандартным соотношением

$$
\chi_{q}(\omega=0)=-\left\langle\left\langle Q_{q} \mid Q_{-q}\right\rangle\right\rangle_{\omega=0}=\frac{1}{\omega_{q}^{2}} \simeq \frac{1}{\Omega_{0}^{2}+c^{2} q^{2}},
$$

где последнее равенство справедливо в дебаевском приближении.

Как можно показать более строго, полученное решение является асимптотически точным для рассматриваемой модели $[5,6]$.

Поскольку уравнение (17) не решается аналитически во всей области температур, рассмотрим его в высокотемпературном и низкотемпературном пределах.

В первом случае $\frac{\omega_{0}}{T} \ll 1$ (здесь $\omega_{0}=c q_{0}=\sqrt{\frac{\Phi(0)}{M}}-$ частота Дебая, $q_{0}-$ дебаевский радиус) уравнение (17) записывается в следующем виде:

$$
M \Omega_{0}^{2}+A=B \frac{T}{N} \sum_{q} \frac{1}{M \Omega_{0}^{2}+\Phi(0)-\Phi(q)} .
$$

Из (18) находим значение критической температуры СФП:

$$
T_{0}=\frac{A \omega_{0}^{2} M}{3 B},
$$


и в наиболее интересном для нас случае низких температур $\frac{\omega_{0}}{T} \exp \left(-\frac{\omega_{0}}{T}\right) \ll 1$ получаем в дебаевском приближении при учете членов порядка не выше $\Omega_{0}^{2}$

$$
\left(g+\frac{1}{2} \ln \frac{\omega_{0}}{T}\right) \Omega_{0}^{2}+\pi T \Omega_{0}-\frac{\pi^{2}}{3}\left(T^{2}-T_{0}^{2}\right)=0,
$$

где $g=\frac{2 M^{2}}{3 B} \omega_{0}^{2}$ и квадрат температуры СФП

$$
T_{0}^{2}=\frac{\omega_{0}}{\pi}\left(2 \frac{A}{B} M \omega_{0}-\frac{3}{2}\right)
$$

Решая квадратное уравнение (20), получаем явное выражение для щели в фононном спектре:

$$
\Omega_{0}=\frac{\pi T}{2 P}\left[\left(1+\frac{4}{3}\left(1-\frac{T_{0}^{2}}{T^{2}}\right) P\right)^{\frac{1}{2}}-1\right],
$$

где обозначено $P=g+\frac{1}{2} \ln \frac{\omega_{0}}{T}$.

В окрестности $T_{0}$ для статической восприимчивости решетки имеем

$$
\chi^{-1}(q=0, \omega=0)=\Omega_{0}^{2} \sim \tau^{\gamma},
$$

здесь введены "безразмерная" температура $\tau=\frac{T-T_{0}}{T_{0}}$ и критический индекс $\gamma$.

Для рассматриваемой модели в области критических флуктуаций $\gamma=2$, когда

$$
\frac{4}{3}\left(g+\frac{1}{2} \ln \frac{\omega_{0}}{T}\right) \frac{T^{2}-T_{0}^{2}}{T^{2}} \ll 1 .
$$

\section{4. ВЛИЯНИЕ КРИТИЧЕСКИХ ФЛУКТУАЦИЙ НА ИЗОТОПИЧЕСКИЙ ЭФФЕКТ}

Обратимся к вычислению температуры сверхпроводящего перехода, воспользовавшись при этом (8). Используя дебаевское приближение для фононного спектра, запишем (8) в виде

$$
\frac{2}{\lambda}=\frac{1}{\omega_{0}} \int_{0}^{\omega_{0}} d \omega \frac{\omega^{2}}{\Omega_{0}^{2}+\omega^{2}} \ln \left[1+\frac{\Omega_{0}^{2}+\omega^{2}}{a^{2} T_{c}^{2}}\right] .
$$

Поскольку этот интеграл точно не берется, ограничимся в расчетах разложением правой части до $\Omega_{0}^{2}$. Таким образом, находим

$$
f(y)+\left(\frac{\Omega_{0}}{\omega_{0}}\right)^{2} g(y)=2\left(1+\frac{1}{\lambda_{0}}\right),
$$

где обозначено

$$
\begin{aligned}
y & =\frac{\omega_{0}}{a T_{c}}, \\
f(y) & =\ln \left(1+y^{2}\right)+\frac{2}{y} \operatorname{arctg}(y), \\
g(y) & =y \operatorname{arctg}(y)-\ln \left(1+y^{2}\right) .
\end{aligned}
$$




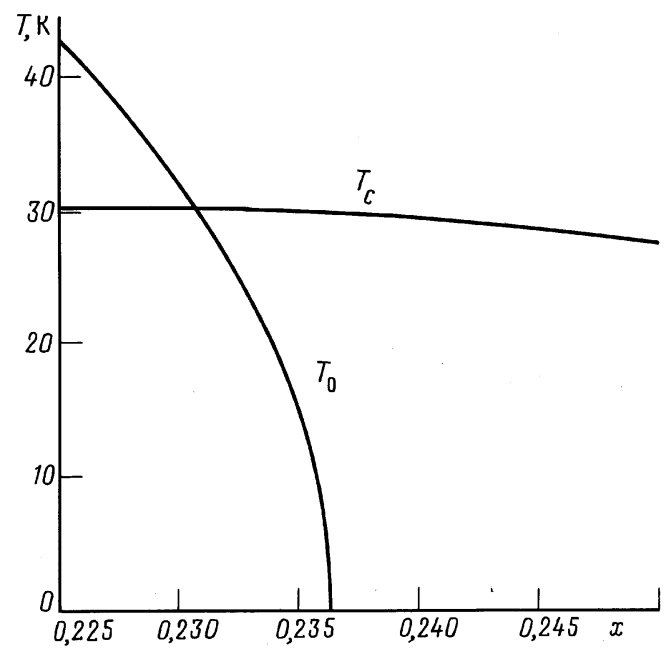

Рис. 1. Фазовая $T-x$-диаграмма для рассматриваемой модели. Верхняя криваятемпература структурного перехода, нижняя - сверхпроводящего в зависимости от концентрации примеси $x$.

Соотношение (24) представляет собой уравнение на температуру $T_{c}$, которая входит в это уравнение не только через величину $y$, но и через зависящую от температуры мягкую моду $\Omega_{0}$. Это уравнение позволяет численно определить $T_{c}$ при заданных параметрах решетки. Будем полагать, что силовая постоянная $A$ зависит от концентрации примесных атомов $x$, как в случае соединений допированного купрата лантана: $A(x)=A_{0}(1-4 x)$ (см. обсуждение в [15]). При некотором значении $x$ температуры сверхпроводящего и структурного переходов совпадают. Окрестность точки пересечения $T_{0}(x)$ и $T_{c}(x)$ на фазовой $T-x$-диаграмме и будет областью критических флуктуаций, поскольку в этой точке $\Omega_{0}=0$ и $T_{c}$ достигает своего максимального значения (см. рис. 1). Это видно непосредственно как из уравнения (24) (поскольку $g(y)>0$, а $f(y) \geq 2$ - возрастающая функция), так и из физических соображений (температура сверхпроводяшего перехода максимальна, если максимальна решеточная восприимчивость).

Рассмотрим ИЭ. Хотя уравнение (24) решается только численно, тем не менее аналитическое выражение для ИЭ может быть получено с помощью дифференцирования его по массе. Рассматривая фононную шель как функцию $\Omega_{0}=\Omega_{0}\left(T_{c}, T_{0}(M), M\right)$, где зависимость от массы через температуру структурного перехода выделена особо, получаем для фактора ИЭ

$$
\alpha=\frac{\frac{1}{2}-\frac{k}{\omega_{0}^{2}}\left(M \frac{\partial \Omega_{0}^{2}}{\partial M}-T_{0} \frac{\partial \Omega_{0}^{2}}{\partial T_{0}} \varepsilon\right)}{1-\frac{k}{\omega_{0}^{2}} T_{c} \frac{\partial \Omega_{0}^{2}}{\partial T_{c}}}
$$

где $k=\frac{g(y)}{y}\left(\frac{\partial f}{\partial y}-\frac{\partial g}{\partial y} \frac{\Omega_{0}^{2}}{\omega_{0}^{2}}\right)^{-1}$ и $\varepsilon=-\frac{\partial \ln T_{0}}{\partial \ln M}-$ ИЭ для температуры СФП. При этом $y$ и $T_{c}$ - решение уравнения (24).

Рассмотрим ИЭ в области критических флуктуаций, где можно положить $\Omega_{0}^{2} \sim \tau^{\gamma}$, 
$\tau=\frac{T_{c}}{T_{0}}-1-$ приведенная температура.

Если $\gamma<1$, то производные квадрата мягкой моды по температуре неограниченно возрастают:

$$
\frac{\partial \Omega_{0}^{2}}{\partial T_{c}} \sim \tau^{\gamma-1} \frac{\partial \tau}{\partial T_{c}} \rightarrow \infty \text { и } \frac{\partial \Omega_{0}^{2}}{\partial T_{0}} \sim \tau^{\gamma-1} \frac{\partial \tau}{\partial T_{0}} \rightarrow \infty \text { при } \tau \rightarrow 0 \quad\left(\text { при этом } \Omega_{0} \rightarrow 0\right),
$$

а производная по массе $\frac{\partial \Omega_{0}^{2}}{\partial M} \rightarrow 0$ при $\tau \rightarrow 0$. Поэтому при $T_{c}=T_{0}$

$$
\alpha=-\varepsilon\left(T_{0} \frac{\partial \tau}{\partial T_{0}}\right) /\left(T_{c} \frac{\partial \tau}{\partial T_{c}}\right)=\varepsilon,
$$

т.е. ИЭ для температуры сверхпроводящего перехода совпадает с ИЭ для структурного перехода. Заметим, что $\varepsilon<0$.

Если $\gamma>1$, то все частные производные от $\Omega_{0}^{2}$ стремятся к нулю при $\Omega_{0} \rightarrow 0$. Поэтому при $T_{c}=T_{0}$ (напомним, что мы положили $\mu^{*}=0$ ) получаем

$$
\alpha=\frac{1}{2}
$$

и, таким образом, в рассматриваемой точке фазовой диаграммы СФП никакого влияния на ИЭ не оказывает.

Полученный результат является общим и не зависит от выбранной модели для СФП. Для рассмотренной в разделе 3 модели с критическим индексом $\gamma=2$ выражение для ИЭ получим, подставляя в (25) значения частных производных от квадрата мягкой моды. Учитывая, что при выбранных параметрах модели (см. ниже) реализуется наиболее интересный случай низких температур $\frac{\omega_{0}}{T} \exp \left(-\frac{\omega_{0}}{T}\right) \ll 1$, из выражения $(22)$ находим ${ }^{1)}$

$$
\begin{aligned}
M \frac{\partial \Omega_{0}^{2}}{\partial M} & =\left(\frac{1}{2}-g\right) \frac{\Omega_{0}^{3}}{D} \\
T_{0} \frac{\partial \Omega_{0}^{2}}{\partial T_{0}} \varepsilon & =-\frac{1}{2}\left(\frac{A}{A_{\mathrm{cr}}}-2\right) \omega_{0}^{2} \frac{\Omega_{0}}{D}, \\
T_{c} \frac{\partial \Omega_{0}^{2}}{\partial T_{c}} & =\frac{\Omega_{0}}{D}\left(\frac{4}{3} \pi^{2} T_{c}^{2}-2 \pi \Omega_{0} T_{c}+\Omega_{0}^{2}\right) .
\end{aligned}
$$

Здесь обозначено $D=2 \Omega_{0} P+\pi T_{c}, A_{\mathrm{cr}}=\frac{3}{4} \frac{B}{M \omega_{0}}-$ значение гармонической постоянной при $T_{0}=0$.

Для численных оценок примем следуюшие значения параметров модели, которые можно сопоставить с экспериментальными данными для купрата лантана $\mathrm{La}_{2-x} \mathrm{Sr}_{x} \mathrm{CuO}_{4}[15]$ :

$$
\omega_{0}=120 \mathrm{~K}, \quad E_{0}=\frac{A^{2}}{4 B}=0,1 \text { эB }, \quad M=16 \text { a.e.м. }, \quad N(0) J^{2}=1 \ni \mathrm{B} / \AA^{2} .
$$

\footnotetext{
${ }^{1)}$ Если выражение для $T_{0}^{2}<0$ (при $A<A_{\mathrm{cr}}$ ), то подразумевается, что
}

$$
T_{0} \frac{\partial \Omega_{0}^{2}}{\partial T_{0}} \varepsilon=\frac{\partial \Omega_{0}^{2}}{\partial T_{0}^{2}} \frac{1}{2} \frac{\partial T_{0}^{2}}{\partial \ln M} .
$$


Здесь $E_{0}$ - высота потенциального барьера для двуямного ангармонического потенциала в недопированном купрате лантана. Предполагается, что от примеси зависит только силовая постоянная $A(x)$. Значение $A_{0}=A(0)$ и $B$ определяются из численного значения $E_{0}$ и уравнения (19) при известной из эксперимента температуре СФП $T_{0}(x=0)=530 \mathrm{~K}$.

Графики зависимости $T_{c}, T_{0}$ и $\alpha$ от концентрации примеси $x$ приведены на рис. 1 и 2. Видно, что $T_{c}$ достигает своего максимального значения, когда $T_{c}=T_{0}$, а $\alpha$ при этом, наоборот, минимально в этой точке фазовой диаграммы.

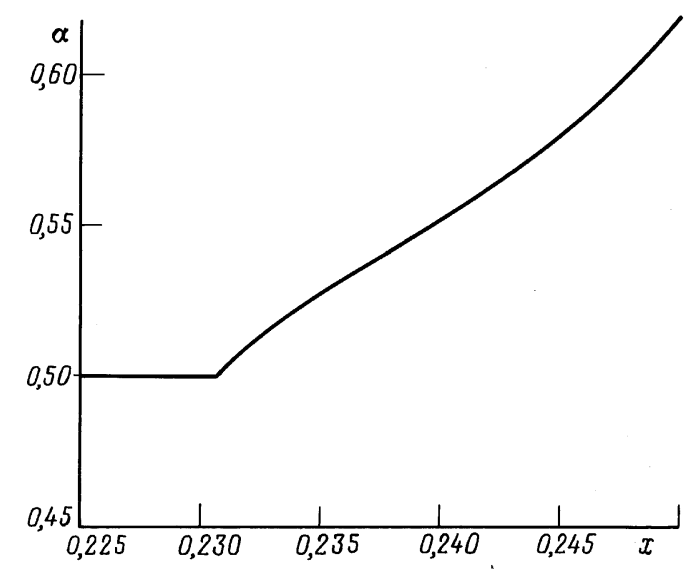

Рис. 2. Зависимость изотопического фактора $\alpha$ от концентрации примеси $x$.

Обсудим полученные результаты. В рамках данной модели СФП критический индекс $\gamma=2$ и влияние критических флуктуаций не столь существенны, как в случае моделей, где $\gamma<1$.

Отметим, что в работах $[3,4]$ мы использовали при расчетах динамики решетки приближение среднего поля, которое дает классический критический индекс $\gamma=1$. Этим обусловлено отличие приведенных там результатов от полученных в этом разделе. Как видно из рис. 2, изотопический фактор $\alpha$ возрастает в высокотемпературной фазе, превышая значение $\frac{1}{2}$. Заметим также, что по мере удаления от температуры СФП значение мягкой моды $\Omega_{0}$ быстро растет и, как показывают численные расчеты, становится сравнимо с дебаевской частотой $\omega_{0}$ уже при $x \geq 0,239$. При этом нарушается сделанное при выводе соотношений (20) и (24) предположение $\frac{\Omega_{0}}{\omega_{0}} \ll 1$, так что для корректных вычислений в этой области концентраций (что не являлось нашей целью) необходимо использовать другие приближения. Из физических соображений следует, что по мере удаления от СФП на $T-x$-диаграмме изотопический фактор будет медленно стремиться к $\frac{1}{2}(\mathrm{~cm} .[3,4])$.

В заключение отметим, что в экспериментах невозможно сколь угодно малое изменение массы изотопа, что фактически подразумевается при расчете изотопического фактора как $\alpha=-\frac{\partial \ln T_{c}}{\partial \ln M}$. При этом на практике изотопический фактор при замене атомов кислорода ${ }^{16} \mathrm{O} \rightarrow{ }^{18} \mathrm{O}$ рассчитывается как

$$
\alpha=-\frac{M}{T_{c}\left({ }^{16} \mathrm{O}\right)} \frac{T_{c}\left({ }^{18} \mathrm{O}\right)-T_{c}\left({ }^{16} \mathrm{O}\right)}{\Delta M}=-8 \frac{T_{c}\left({ }^{18} \mathrm{O}\right)-T_{c}\left({ }^{16} \mathrm{O}\right)}{T_{c}\left({ }^{16} \mathrm{O}\right)} .
$$


В этом случае, если при изотопической замене приращение температуры сверхпроводяшего перехода превышает характерный размер области критических флуктуаций, то соотношения (26) и (27) могут и не выполняться.

Автор хотел бы выразить благодарность профессору Н. М. Плакиде за многочисленные обсуждения и поддержку.

\section{Список литературы}

[1] Frank J. P. // Physical Properties of High-Temperature Superconductors. V.4 / Ed. D. M. Ginsberg. Singapore: World Scientific, 1994. P. 189.

[2] Plakida N.M., Aksenov V.L., Drechsler S. L. // Europhys. Lett. 1987. V. 4. P. 1309-1314.

[3] Плакида Н. М., Черный А. Ю. // Сверхпроводимость ФХТ. 1991. Т. 4. С. 1493.

[4] Cherny A. Yu., Galbaatar T. // Physica. 1993. V. C 210. P. 35-41.

[5] Плакида Н. М., Тончев Н. С. // ТМФ. 1985. Т. 63. №2. С. 270-279.

[6] Плакида Н. М., Тончев Н. С. Препринт ОИЯИ Р17-85-400. Дубна: ОИЯИ. 1985.

[7] Плакида Н. М., Черный А. Ю. // ТМФ. 1990. Т. 84. №1. С. 120-127.

[8] Боголюбов Н. Н., Толмачев В. В., ШІирков Д. В. Новый методв теории сверхпроводимости. М.: Наука, 1977.

[9] Вуйчич Г. М., Петру З. К., Плакида Н. М. // ТМФ. 1981. Т. 46. №1. С. 91-98.

[10] Зубарев Д. Н. Неравновесная статистическая термодинамика. М.: Наука, 1971.

[11] McMillan W. L. // Phys. Rev. 1968. V. 167. P. 331-344.

[12] Брус А. Д., Каули Р. А. Структурные фазовые переходы. М.: Мир, 1984.

[13] Allen P. B., Mitrović B. // Solid State Physics / Eds. Seitz F. and Turnbull D.. V. 37. N.Y.: Academic, 1982. P. 1-92.

[14] Combescot $R$. // Труды международного семинара по ВТСП. Дубна 3-6 июля, 1990. Изд. ОИЯИ Е-17-90-472. Дубна: ОИЯИ, 1990. С. 68-82.

[15] Flax S., Plakida N. M., Aksenov V. L. // Int. J. Mod. Phys. 1990. V. 4. P. 1955-1973.

[16] Плакида Н. М. // Статистическая физика и квантовая теория поля / Под ред. Н. Н. Боголюбова. М.: Наука, 1973. С. 205-240.

Объединенный институт

ядерных исследований

Поступила в редакцию 22.V.1995 г

\section{A. Yu. Cherny \\ THE INFLUENCE OF THE CRITICAL FLUCTUATIONS UNDER THE STRUCTURAL PHASE TRANSITION ON THE SUPERCONDUCTING PAIRING}

The influence of the critical fluctuations in the vicinity of the structural phase transition (SPT) on the isotope effect (IE) is studied within the anharmonic model of the high-temperature superconductivity. It is shown that if the superconducting transition temperature $T_{c}$ is equal to the temperature of SPT, then the IE is determined by the critical exponent $\gamma$. In the case $\gamma<1$ the isotope factor for $T_{c}\left(\alpha=-\frac{\partial \ln T_{c}}{\partial \ln M}\right)$ is equal to one for the structural transition temperature $T_{0}$ (i.e. $\alpha=\varepsilon$, where $\varepsilon=-\frac{\partial \ln T_{0}}{\partial \ln M}$ ), but in the case $\gamma>1 \alpha=\frac{1}{2}$ (if one neglects Coulomb interaction). This result does not depend on the model for the SPT. The result is demonstrated by considering the exactly solvable model of the SPT with $\gamma=2$. 